\title{
A new method for significant wave height retrieval from ScanSAR mode imagery
}

\author{
Lin Ren, Jingsong Yang, Weiyong Shi \\ State Key Laboratory of Satellite Ocean Environment \\ Dynamics, \\ Second Institute of Oceanography, SOA \\ 36 Baochubei Road, Hangzhou, 310012, China \\ renlin210@sio.org.cn
}

\author{
Jun Zhang \\ Third Institute of Oceanography, SOA \\ 178 Daxue Road, Xiamen, 361005, China
}

\begin{abstract}
We usually make use of the wave stripes of the synthetic aperture $\operatorname{radar}(\mathrm{SAR})$ imagery to retrieve the ocean wave properties based on the SAR-ocean mapping model. Due to the lower resolution, the ScanSAR mode imagery has no apparent ocean wave stripes but possesses the great advantage of large area of observation. To exert the advantage of ScanSAR imagery, this paper develops the new model to retrieve the significant wave height(SWH) in case of no wave stripes. Firstly, we collect the isochronous wind speed and SWH from buoy according to the ScanSAR imagery. Then we propose the mode expression and debug out the coefficient by the fitting based on the isochronous data. Finally, we use other isochronous data to validate the model. Retrieved SWH have a correlation coefficient of 0.83 and root mean square error(rmse) of $0.33 \mathrm{~m}$ with the $\mathrm{SWH}$ of buoy. It shows the ScanSAR imagery has the ability of retrieving SWH.
\end{abstract}

Index Terms-ScanSAR imagery, retrieval, significant wave height.

\section{INTRODUCTION}

For high resolution synthetic aperture radar(SAR) imagery, it can shows the wave stripes and retrieve the ocean wave spectra based on the nonlinear SAR-ocean mapping model. Then any wave parameters like significant wave height(SWH) can be extracted from the spectra [1-6]. The above method is complex and only aims at the imagery having wave stripes. Due to the lower resolution(approximately $80 \mathrm{~m}$ ), the ScanSAR mode imagery has no apparent ocean wave stripes so that it can't retrieve SWH from the method. But ScanSAR imagery possesses the great advantage of large area of observation. As we known, SAR can retrieve the wind speed with empirical geophysical model function(GMF) between the backscatter coefficient(sigma0) and wind speed [7]. This paper will try similar method to develop the direct empirical model between the sigma0 and SWH. From the mode, we can extract the SWH of SAR imagery in case of no wave stripes and prove the status of ocean environment monitoring.

\section{DATA}

The isochronous data collected in this paper include: (1) ScanSAR imagery. We select ENVISAT ASAR WSS mode data as the ScanSAR imagery, which has the swath of $400 \mathrm{~km}$ $\times 400 \mathrm{~km}$ and the resolution of $80 \mathrm{~m} \times 7.8 \mathrm{~m}$ (Azimuth direction $\times$ Range direction). (2) Buoy data. It can provide the accurate SWH and wind speed data. Part of collected data are used to develop the mode of SWH retrieval from ScanSAR imagery, and the other part are used to validate the model performance.

The time of data is from January 1, 2009 to December 31, 2012. The location of data focus on the South China Sea(SCS). Figure 1 shows the coverage area of ScanSAR imagery. Based on the location of ScanSAR imagery, we collect the isochronous wind speed and SWH data from the buoy. The isochronous temporal window is 3 hour and spatial window is $25 \mathrm{~km}$ 


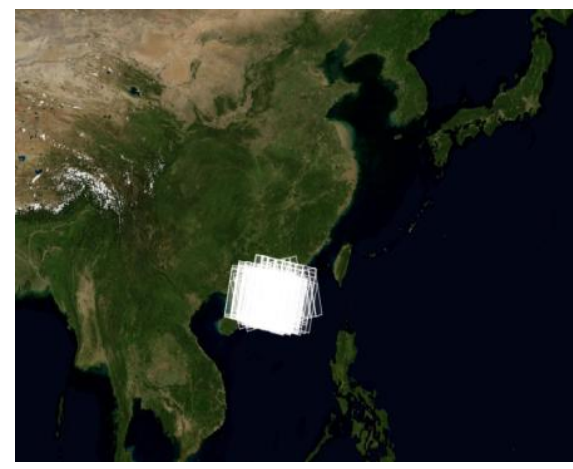

Fig. 1. The coverage area of all ScanSAR imagery, the white pane are the edges of imagery

\section{DATA PROCESSING}

\section{A. SAR imagery preprocessing}

Figure 2 describes the preprocessing flow of ENVISAT ASAR WSS imagery. The main step is as follow:

(1)Image segmentation

ASAR WSS imagery has the wide swath, which is acquired from the combination of five antenna beams(SS1 to SS5) and is delivered as a single data file containing the subswath data records arranged sequentially. The five WSS beams acquire data with a substantial overlap (typically several hundred range samples, $\sim 9 \mathrm{Km}$ ). So we must spilt it into five imagery and eliminate the overlapping portion.

\section{(2)Deburst}

As the ScanSAR imagery, WSS imagery work at burst mode. The imagery has many overlapping 'bursts' along the flight direction associated with a zero Doppler time for each range line. Figure 3 shows the discontinuous signal due to overlapping 'bursts'. This paper will eliminate the discontinuity with the average method.

\section{(3) Calibration}

The pixel intensity of image can be converted into sigma0 with the following equation [8].

$$
\sigma_{i, j}^{o}=\frac{\mathrm{DN}_{i, j}^{2}}{K} \cdot \frac{q}{G\left(\theta_{i, j}\right)^{2}}\left(\frac{R_{i, j}}{R_{r e f}}\right)^{3} \sin \left(\alpha_{i, j}\right)
$$

Where $\sigma_{i, j}^{o}$ is sigma0 at image, $K$ is absolute calibration constant, $\mathrm{DN}_{i, j}$ is the pixel intensity at image line and column "i,j", $G\left(\theta_{i, j}\right)$ is the two-way antenna gain at the look angle corresponding to pixel " $\mathrm{i}, \mathrm{j}$ ", $R_{i, j}$ is the slant range distance to pixel line and column "i,j", $R_{\text {ref }}$ is the reference slant range distance, $\alpha_{i, j}$ is the incidence angle at pixel line and column "i,j".

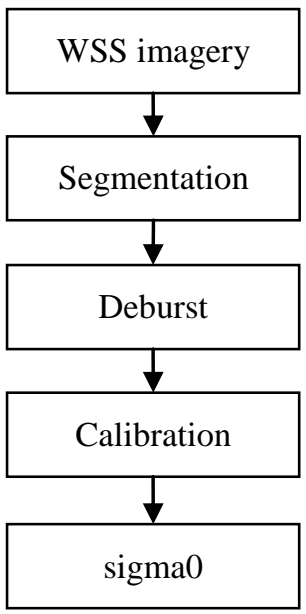

Fig. 2. The preprocessing flow of ENVISAT ASAR WSS imagery

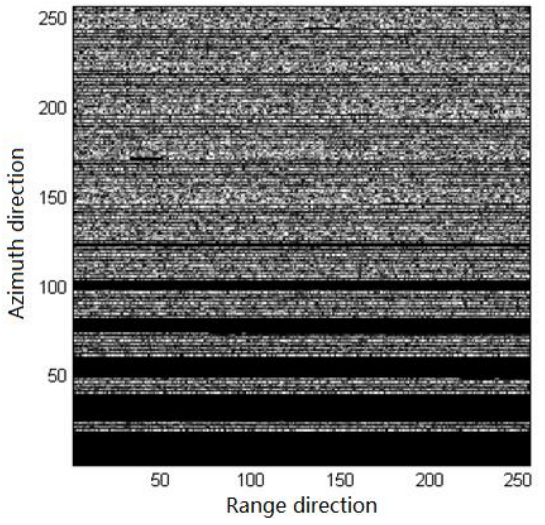

Fig. 3. The original ENVISAT ASAR WSS imagery

\section{B. Wind speed height correction}

Because the wind speed data from buoy are measured on different height above the sea surface. Therefore, we convert them into same height of $10 \mathrm{~m}$ by the following equation [9].

$$
U_{10}=\left[\ln (10 / z) / \ln \left(H_{\text {bиоу }} / z\right)\right] \times U\left(H_{\text {bиоу }}\right)
$$


Where $U_{10}$ is the wind speed for $10 \mathrm{~m}, z$ is roughness length and set to be $1.52 \times 10^{-4}, H_{\text {buoy }}$ is the measurement height of buoy.

\section{MODEL}

In this paper, we develop a model to retrieve the SWH from ScanSAR imagery. Equation 3 is the proposed model expression, which has 9 coefficients. Figure 4 is the chart flow of establishing the model among the SWH, wind speed and sigma0. The model coefficients are debugged out by multi-parameters fitting and listed in Table 1.

$$
\begin{aligned}
S W H= & x 0+x 1 \cdot \sigma_{0}+x 2 \cdot \sigma_{0}^{2}+ \\
& \times 3 \cdot U_{10}+x 4 \cdot U_{10}^{2}+x 5 \cdot \sigma_{0} \cdot U_{10}+ \\
& \times 6 \cdot \sigma_{0}^{2} \cdot U_{10}+x 7 \cdot \sigma_{0} \cdot U_{10}^{2}+x 8 \cdot \sigma_{0}^{2} \cdot U_{10}^{2}
\end{aligned}
$$

Where $\sigma_{0}$ is the mean sigma 0 for the area of $540 \mathrm{~m} \times 540$ $\mathrm{m}, \mathrm{x} 0-\mathrm{x} 8$ is the constant coefficient.

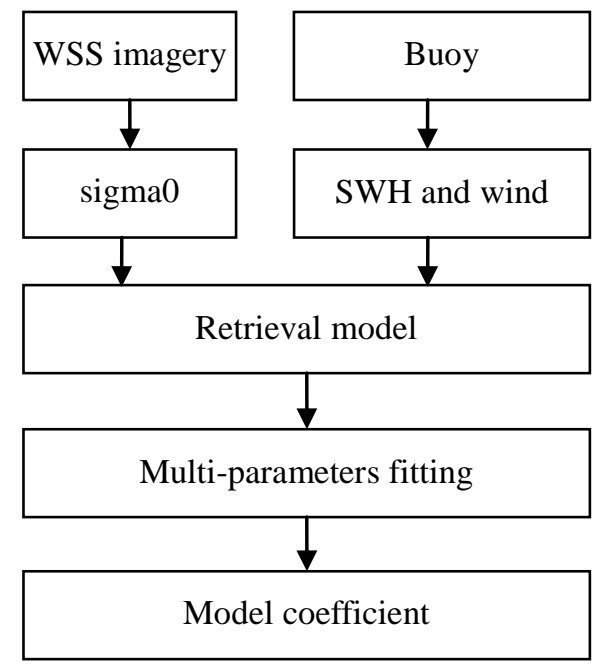

Fig. 4. The chart flow of establishing the retrieved model

TABLE I. MODEL COEFFICIENT

\begin{tabular}{cccc}
\hline Coefficient & Value & Coefficient & Value \\
\hline $\mathbf{x 0}$ & 1.5975 & $\mathbf{x 5}$ & 0.7698 \\
$\mathbf{x 1}$ & -1.8179 & $\mathbf{x 6}$ & -0.3943 \\
$\mathbf{x} \mathbf{6}$ & 1.0161 & $\mathbf{x 7}$ & -0.0679 \\
$\mathbf{x 3}$ & -0.3101 & $\mathbf{x 8}$ & 0.0342 \\
$\mathbf{x 4}$ & 0.0394 & / & / \\
\hline
\end{tabular}

\section{VALIDATION}

In this section, the other isochronous WSS imagery and buoy data are used to validate the model performance. Firstly, we convert the imagery into sigma0 by the preprocessing. Then the wind speed are retrieved from the CMOD_IFR2 model. Finally, the sigma0 and wind speed are imported into the developed model to retrieve the SWH. Figure 5 is the scatter of SWH from the WSS imagery and the buoy. It shows the correlation coefficient of 0.83 . Figure 6 is the comparing of SWH from the WSS imagery and the buoy. They have the rmse of $0.33 \mathrm{~m}$ and relative error of $17.2 \%$.

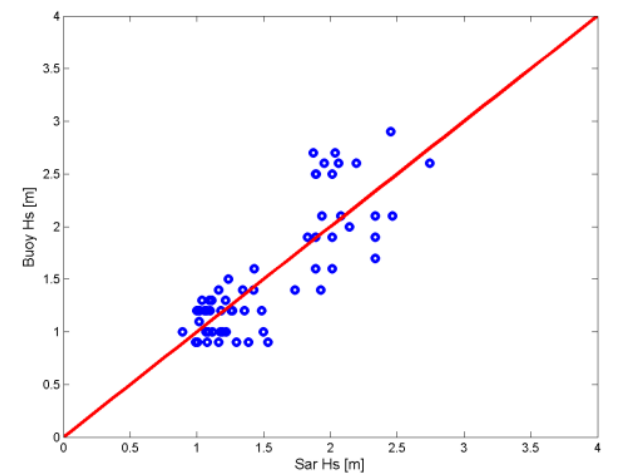

Fig. 5. The scatter of SWH from the WSS imagery and the buoy

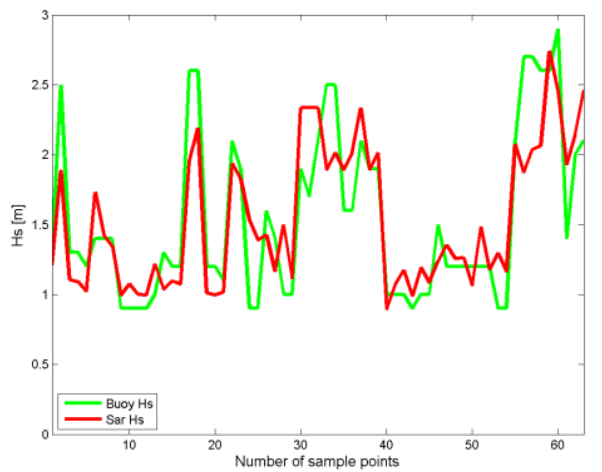

Fig. 6. The comparing of SWH from the WSS imagery and the buoy

\section{CONCLUSION}

This paper aims to develop the SWH retrieval model from the ScanSAR mode imagery. Firstly, we collect the isochronous WSS imagery and buoy data. Buoy provide the wind speed and SWH data. Then we propose the model expression and debug out the 9 model coefficients. Finally, we use the developed model to retrieve the SWH from the imagery. The retrieved SWH are compared with the ones of buoy. 
Comparative results show it has the correlation coefficient of 0.83 , rmse of $0.33 \mathrm{~m}$ and relative error of $17.2 \%$ on SWH from the WSS imagery and the buoy. It demonstrates ScanSAR imagery has the ability of retrieving the SWH.

\section{ACKNOWLEDGMENT}

Partly granted by the Marine renewable energy special fund project (GHME2011ZC05 and GHME2011ZC06).

\section{REFERENCES}

[1] Hasselmann K, Hasselmann S. On the nonlinear mapping of an ocean wave spectrum into a synthetic aperture radar image spectrum and its inversion. J. Geophys. Res.. 1991, 96(C6): 10713-10729.

[2] Engen G, Johnsen H. SAR-ocean wave inversion using image cross spectra. IEEE transactions on geoscience and remote sensing, 1995, 33(4): 1047-1056.

[3] Mastenbroek C, Valk C F. A semiparametric algorithm to retrieve ocean wave spectra from synthetic aperture radar. J. Geophys.
Res., 2000, 105(C2): 3497-3516.

[4] Collard F, Ardhuin F, Chapron B. Extraction of coastal ocean wave fields from SAR images. IEEE Journal of Oceanic Engineering, 2005, 30(3): 526-533.

[5] Sun J, Guan C. Parameterized first-guess spectrum method for retrieving directional spectrum of swell-dominated waves and huge waves from SAR images. Chinese Journal of Oceanology and Limnology, 2006, 24(1): 12-20.

[6] Ren L, Yang J S, Chen P. A method for retrieving the directional ocean wave spectra from synthetic aperture radar image. Proceedings of SPIE. United Kingdom, 2012:85320Z.

[7] Long A. C-band V-polarized radar sea-echo model from ERS-1 Haltenbanken campaign. ERS-1 Geophys Validation Workshop, 1995.

[8] ESA. ASAR Product Handbook. 2007.

[9] Wentz F, et al. Measurement of oceanic wind vector using satellite microwave radiometers. IEEE Transactions on Geoscience and Remote Sensing. 1992, 30(5): 960-97. 